\title{
Polimorfismo S19W (Ser19Ter) de la APOA5 y su relación con la hipertrigliceridemia en una población de Colombia
}

\author{
S19W polymorphism (Ser19Ter) of APOA5 and its relationship \\ with hypertriglyceridemia in a Colombian population
}

Gerardo Mantilla $\mathrm{M}^{1}$, Adriana Castillo $\mathrm{P}^{1}$, Viviana L. Pérez $\mathrm{F}^{1,2}$, Clara I. Vargas $\mathrm{C}^{1}$, Fernando Rondón $\mathrm{G}^{1}$

Forma de citar: Mantilla G, Castillo A, Pérez V, Vargas C, Rondón F. Polimorfismo S19W (Ser19Ter) de la APOA5 y su relación con la hipertrigliceridemia en una población de Bucaramanga, Colombia. Rev Univ Ind Santander Salud. 2017; 49(1) 29-35. DOI: http://dx.doi.org/10.18273/revsal.v49n1-2017003 @c) (1)

\section{RESUMEN}

Introducción: Las enfermedades cardiovasculares (ECV) son responsables del 29,69\% de las muertes en Colombia. Se ha encontrado la hipertrigliceridemia, en diversos estudios como factor de riesgo independiente para la ECV. El polimorfismo S19W (Ser19Ter) de la ApoA5 se ha asociado en algunas poblaciones con la hipertrigliceridemia. Sin embargo, en Colombia esto ha sido poco estudiado. Objetivo: Estimar la asociación entre el polimorfismo S19W y la hipertrigliceridemia en población colombiana. Metodología: Estudio tipo Corte Transversal, con 400 individuos provenientes de Bucaramanga, Colombia. Se cuantificó los TAG y se genotipificaron mediante la técnica de SNaPshot y mini secuenciación. Los resultados fueron analizados utilizando el software de análisis genético Arlequín 3.5.1.2. Resultados: El polimorfismo S19W (Ser19Ter) mostró tres perfiles, CC, GG y CG. El polimorfismo S19W se caracterizó tanto en afectados como en no afectados, mostrando que no existen diferencias significativas en esta distribución cuando se comparan los dos grupos. Discusión: Diversos mecanismos se han propuesto para sustentar la hipertrigliceridemia como un factor de riesgo para ECV, entre los que se cuenta la APOA5. El estudio comprobó que la población estudiada se encuentra en equilibrio de Hardy Weinberg y al genotipo CC como el más frecuente. Los genotipos GG y el GC presentaron valores significativos en el grupo de sujetos afectados, ( $p<0,01$ y $\mathrm{p}=0,03$; respectivamente). Se demostró la existencia de una estrecha relación entre el polimorfismo Ser19Trp y la hipertrigliceridemia $(\mathrm{p}<0,01)$. Conclusión: Se pudo demostrar la existencia de una relación entre el polimorfismo Ser19Trp de la Apo A5 con los niveles elevados de TAG $(\mathrm{p}<0,01)$.

Palabras claves: Polimorfismo genético, hipertrigliceridemia, cardiopatía isquémica, Apo A.

\begin{abstract}
Introduction: Cardiovascular diseases (CVD) are responsible for $29.69 \%$ of the deaths in Colombia. Several studies have shown that hypertriglyceridemia is an independent risk factor for CVD. ApoA5 gene S19W (Ser19Ter) polymorphism has been associated with hypertriglyceridemia in some populations; however, their

1. Universidad Industrial de Santander. Bucaramanga, Colombia.

2. Universidad de Santander. Bucaramanga, Colombia.

Correspondencia: Gerardo Mantilla M. Dirección: Carrera 32 № 29 -31. Correo electrónico gmanti@uis.edu.co.Teléfono: +7 6344000 ext. 3131


influence in Colombia is unknown. Objective: To determine the relationship between S19W polymorphism and hypertriglyceridemia in Colombian population. Methodology: Transversal crossover Studio, included a total of 400 individuals. TAG was quantified and genotyped using the technique SnapShot and mini sequencing. The results were analyzed using genetic analysis software Arlequin 3.5.1.2. Results: S19W (Ser19Ter) polymorphism showed three profiles, CC, GG and CG. The S19W polymorphism was characterized both affected and not affected. There wasn't significant differences in the distribution when the two groups are compared. Discussion: Various mechanisms have been proposed to support hypertriglyceridemia as a risk factor for CVD, including the APOA5 counted. The study found that the study population is in Hardy Weinberg and CC genotype as the most frequent. The GG and GC genotypes showed significant values in the group of affected subjects $(p=0.002$ and 0.03$)$. It demonstrated the existence of a close relationship between the Ser19Trp polymorphism and hypertriglyceridemia $(\mathrm{p}<0,01)$. Conclusion: It was possible to demonstrate the existence of a relationship between polymorphism Ser19Trp of ApoA5 with elevated levels of TAG $(\mathrm{p}<0,01)$.

Keywords: Cardiovascular disease, polymorphism genetic, Hypertriglyceridemia, Cardiovascular Diseases, APOA5 protein, human

\section{INTRODUCCIÓN}

En la actualidad, el infarto, la trombosis, la diabetes y la hipertensión se cuentan entre las 10 primeras causas de muerte en Colombia. Durante el periodo 2005 - 2011, las enfermedades cardiovasculares (ECV) fueron la primera causa de muerte en la población colombiana, con $29,69 \%$ del total de muertes, siendo una de las primeras causas de discapacidad y pérdida laboral ${ }^{1}$. El origen de estas patologías es multifactorial, con un componente genético y factores medio-ambientales que interactúan contribuyendo a la susceptibilidad individual a estas enfermedades.

Diversos estudios epidemiológicos y clínicos han logrado demostrar que los niveles elevados de triglicéridos (TAG), son un factor de riesgo independiente para $\mathrm{ECV}^{2,3}$. Estos niveles están regulados por factores genéticos y medio-ambientales, produciendo variabilidad en la población ${ }^{4}$. Una molécula involucrada en el metabolismo de los TAG es la lipoproteína lipasa (LPL), enzima que se encarga de la hidrólisis de los TAG transportados por la VLDL y el quilomicrón hasta ácidos grasos libre y glicerol, con el objetivo de aportar sustrato energético a los tejidos periféricos $^{5}$. La LPL es sintetizada por los tejidos periféricos y liberada a la circulación, posteriormente se ancla al endotelio vascular adyacente unido al heparán sulfato ${ }^{6,7}$. Para llevar a cabo su actividad catalítica sobre las lipoproteínas ricas en TAG, la LPL necesita de varias moléculas entre las que se cuentan APOA5 y APOCII que permiten el anclaje y actividad de la enzima sobre los TAG presentes en las lipoproteínas ${ }^{6,7}$.

La APOA5 es una proteína de la familia de las apoproteínas, formada por 366 aminoácidos, con un peso molecular de $39 \mathrm{kDa}$. Es sintetizada en el hígado para luego aparecer en circulación asociada como dímero a lipoproteínas ricas en TAG como la VLDL, así como en la HDL. Aunque su mecanismo no es claro, funciona como activador de la LPL, contribuyendo al control de los niveles de TAG circulantes, presentes en el quilomicrón y la VLDL. El gen de la APOA5 hace parte del clúster APOA1/CIII/AIV localizado en el cromosoma 11 región q23 y está formada por cuatro exones que codifican para un marco de lectura de $1107 \mathrm{pb}^{7}$. Cuatro polimorfismos de la APOA5 se han reportado asociados de manera diferencial a niveles anormales de TAG en humanos en diversos grupos étnicos ${ }^{8,9}$. El polimorfismo S19W (rs 3135506), incluye la sustitución de una citosina por una guanina en el nucleótido 56 del codón 19, lo que resulta en el cambio de una serina por un triptófano ${ }^{10}$, ocasionando la disminución de la actividad de la APOA5; esto lleva a una hipertrigliceridemia marcada y a un riesgo mayor para $E C V^{10,11}$. Sin embargo, existen reportes sobre la no relación del polimorfismo S19W de la APOA5 con la ECV, por tanto, esta asociación no está del todo determinada ${ }^{12}$. La prevalencia de este polimorfismo ha sido estimada en un 6\% en individuos caucásicos, 7\% en afroamericanos y $15 \%$ en hispanos ${ }^{13-15}$.

Hasta el momento no se ha reportado la prevalencia de este polimorfismo y su relación con la hipertrigliceridemia en la población colombiana. Si se tiene en cuenta las diferencias dietarias que caracterizan nuestra cultura y la variabilidad genética producto del mestizaje, la influencia de este polimorfismo puede ser diferente para la población colombiana en relación al resto del mundo. Este estudio tuvo como objetivo explorar la relación existente en población colombiana que porta el polimorfismo S19W (Ser19Ter) y la 
hipertrigliceridemia, como un aporte inicial al estudio del impacto genético en el metabolismo de los lípidos.

Estudio tipo Corte Transversal, que incluyó 400 individuos con edades entre 19 y 69 años, colombianos, residentes en Bucaramanga, participantes en los estudios CARMEN (Conjunto de Acciones para la Reducción Multifactorial de Enfermedades Crónicas no Trasmisibles), e INEFAC (Incidencia de Enfermedades Cardiovasculares y de sus Factores de Riesgo), la muestra se seleccionó al azar a partir de la base de datos de CARMEN e INEFAC. Después de explicar las condiciones del ensayo y haber firmado un consentimiento informado, cada participante fue sometido a un periodo de ayuno de 10 a 12 horas, para posteriormente tomarle una muestra de sangre en tubo seco utilizada para el análisis bioquímico y en tubo EDTA como anticoagulante, para el análisis genético. El tubo seco fue centrifugado a $3000 \mathrm{rpm}$ durante 15 minutos. El suero obtenido fue alicuotado y almacenado a $-20^{\circ} \mathrm{C}$ hasta el momento de su análisis.

El proceso de análisis molecular, se inició con la extracción de ADN utilizando el kit ProFiler Forensic DNA $^{\circledR}$. Para la identificación del polimorfismo S19W (Ser19Trp) se amplificó un fragmento de $268 \mathrm{pb}$ mediante técnica de PCR, utilizando como cebadores 5'-CCAGCTGTCTCCTCCCTTC-3'(F) y 5'-TCTCCGACCCTGACTTCAAC-3'(R). La identificación del polimorfismo se efectuó mediante mini secuenciación del fragmento amplificado, empleando un kit de SNaPshot Multiplex ${ }^{\circledR}$ (Applied Biosystems), utilizando un secuenciador ABI PRISM $^{\circledR} 310$ (Applied Biosystems). Los tres perfiles característicos, se identificaron en el electroferograma por el color del marcador utilizado: negro para guanina (G), azul para citosina (C) y un patrón mixto para el heterocigoto guanina citosina (GC). Para cuantificar la concentración de triacilgliceridos en la muestra se utilizó la técnica colorimétrica, enzimática, GPO/POD (Wiener). Se consideró hipertrigliceridemia cuando los niveles plasmáticos de TAG son superiores a 150 $\mathrm{mgr} / \mathrm{dL}$.

El análisis de los datos obtenidos se realizó utilizando el programa de análisis genético poblacional Arlequín 3.5.1.2 ${ }^{16}$; para las variables continuas se calculó la media \pm desviación estándar. Además, se utilizó el test de $\mathrm{Chi}^{2}\left(\mathrm{X}^{2}\right)$ para el análisis de las variables no continuas y para verificar el equilibrio HardyWeinberg (EHW), el cual permitió corroborar que los individuos se aparearon al azar.

El control de calidad de los ensayos moleculares se hizo incluyendo en todas las pruebas controles positivos y negativos (Figura 1); la correcta genotipificación fue confirmada mediante la repetición al azar de un $20 \%$ de las muestras analizadas.
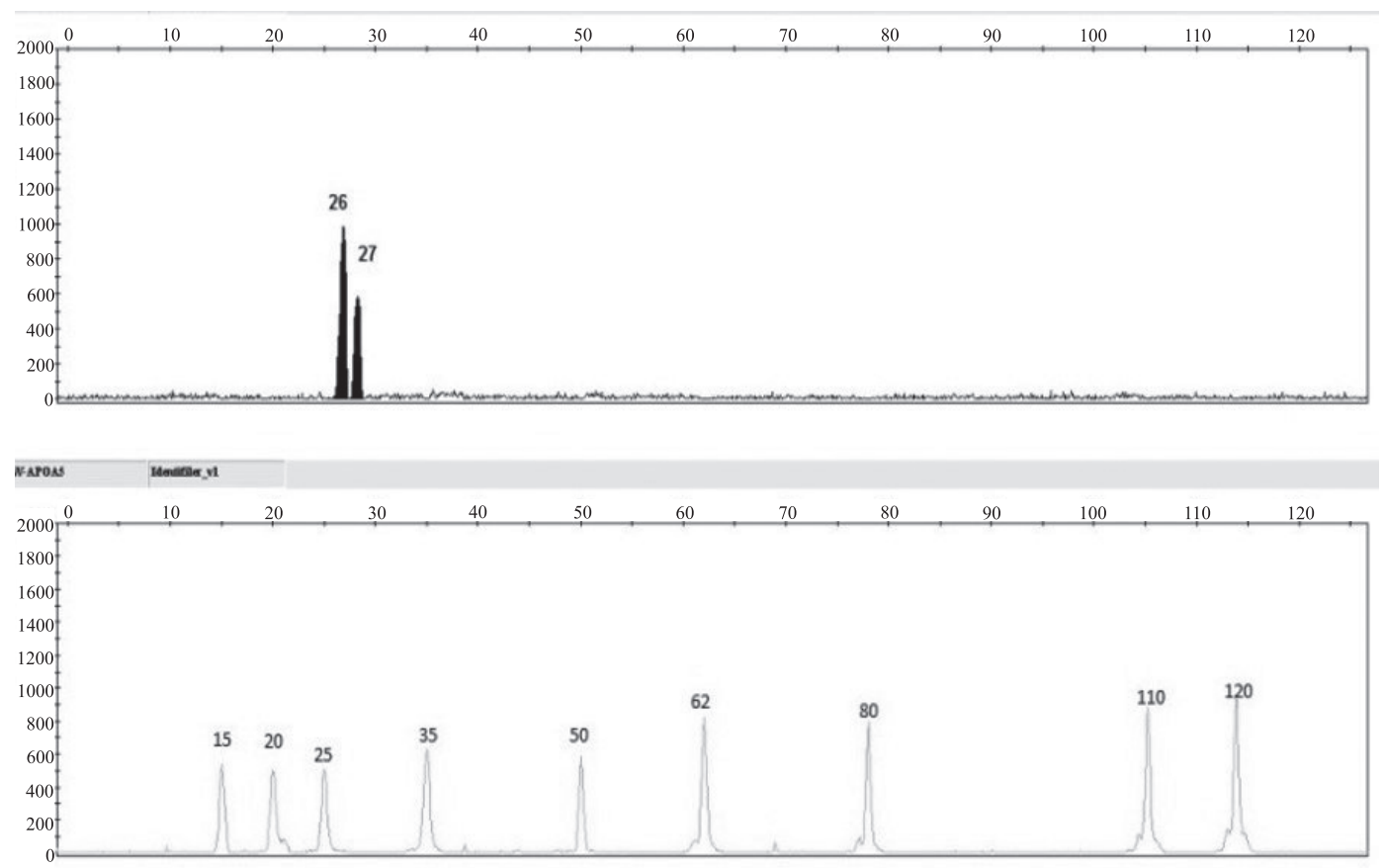

Figura 1. Control positivo polimorfismo S19W (Ser19Ter). En el recuadro A se muestra los dos picos del control de 26 y $27 \mathrm{pb}$ (color negro), marcados con el fluorocromo amarillo. En el recuadro B se observa en color naranja, la imagen del marcador de peso molecular (The GeneScan ${ }^{\mathrm{TM}} 120 \mathrm{Lyz}^{\circledR}$ size standard). 


\section{RESULTADOS}

El estudio incluyó 400 participantes, 272 (68\%) hombres con edad promedio de 37,6 años. El 39,8\% de los participantes (159) presentaron hipertrigliceridemia, mientras que en el 60,2\% (241) se encontraron niveles normales (Tabla 1). Se determinó que los grupos analizados se encuentran en equilibrio de Hardy Weinberg. (Afectado $\mathrm{X}^{2}=1,53$; no afectado $\mathrm{X}^{2}=0,58$ ) lo que permitió inferir que los mismos representan la población en general.

El polimorfismo S19W (Ser19Ter) mostró tres perfiles, dos genotipos homocigotos CC y GG y uno heterocigoto CG. El genotipo CC correspondiente al mutado, presenta un pico de $27 \mathrm{pb}$, se encontró en el $2 \%$ de la muestra, y el genotipo GG correspondiente al ancestral, exhibe un pico de $26 \mathrm{pb}$, se encontró en el $73.4 \%$ del grupo analizado. El perfil heterocigoto CG se caracteriza por un pico 26 y otro de $27 \mathrm{pb}$, se detectó en el $24.6 \%$ de la población analizada (Figura 2).

Tabla 1. Concentraciones séricas de triglicéridos (TAG) de acuerdo con los genotipos de los sujetos afectados y no afectados.

\begin{tabular}{|c|c|c|c|}
\hline Genotipo & $\begin{array}{c}\text { TAG }^{\mathrm{a}}<150 \mathrm{mg} / \mathrm{dL} \\
(\mathrm{No} \text { afectados) } \\
\mathbf{n}=\mathbf{2 4 1}(\mathbf{6 0 . 2 \%})\end{array}$ & $\begin{array}{c}\text { TAG } \geq 150 \mathrm{mg} / \mathrm{dL} \\
\quad(A f e c t a d o s) \\
n=159(39.8 \%)\end{array}$ & $\begin{array}{c}\text { Total } \\
N=400 \\
(100 \%)\end{array}$ \\
\hline $\mathrm{CC}$ & $3(1)$ & $6(3.8)$ & $9(2.25)$ \\
\hline $\mathrm{CG}$ & $59(24.5)$ & $38(23.9)$ & $97(24.25)$ \\
\hline GG & $179(74.3)$ & $115(72.3)$ & $294(73.5)$ \\
\hline
\end{tabular}

Pearson $\operatorname{chi}^{2}(2)=2.7854 \mathrm{p}=0,25$

Fisher's exact $=\quad \mathrm{p}=0,29$

(a)

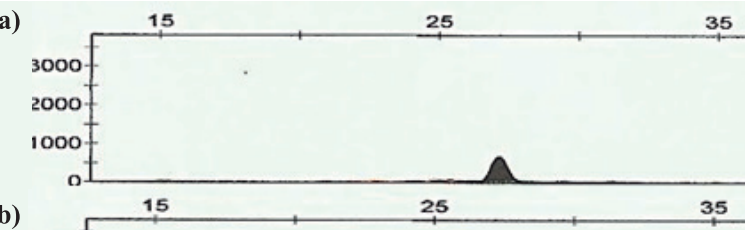

(b)

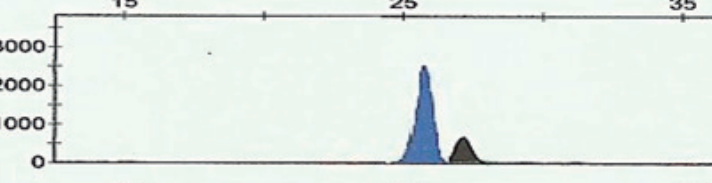

(c)

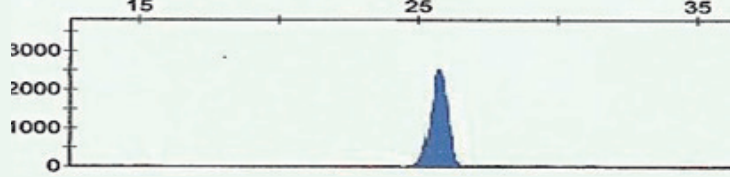

Figura 2. Electroferograma obtenido con los perfiles genéticos del amplificado del polimorfismo Ser19Trp de la ApoA5. La figura (a) muestra el perfil CC (pico en $27 \mathrm{pb}$ ); (b) el perfil GC (picos en 26 y $27 \mathrm{pb}$ ) y (c) el perfil GG (picos en $26 \mathrm{pb}$ ).
La distribución de los genotipos del polimorfismo S19W (Ser19Ter) se estableció para sujetos afectados con hipertrigliceridemia (TAG $\geq 150 \mathrm{mg} / \mathrm{dL}$ ) y no afectados. El genotipo GG fue el más frecuente en la población estudiada, tanto en afectados como no afectados, representando el $73,5 \%$ de la población estudiada, mientras el genotipo CC fue el menos frecuente para ambos grupos de individuos, representando solo el 2,2\% de la población. No existen diferencias significativas en esta distribución cuando se comparan los dos grupos, (valor $\mathrm{X}^{2}=2.7854, \mathrm{p}=0.248$ ). (Tabla 2).

Al comparar en la muestra analizada la distribución genotípica y la alélica, se encontró la forma ancestral del polimorfismo S19W (Ser19Ter), correspondiente a la forma homocigota GG, en el grupo de afectados, con un valor significativo $(p=0,002)$, igualmente se observó para la forma heterocigota $\mathrm{CG}(\mathrm{p}=0.033)$, en los dos grupos analizados. Este hallazgo se correlaciona con lo observado en la distribución alélica de la muestra analizada, para el alelo $\mathrm{G}$ en el grupo de afectados el valor de $\mathrm{p}$ fue inferior a $\mathrm{p}<0,01$. Las dos poblaciones, afectados y no afectados mostraron una distribución diferencial. (Tabla 2).

Al explorar la posible relación entre el polimorfismo Ser19Trp de la ApoA5 con los niveles elevados de TAG $(\geq 150 \mathrm{mg} / \mathrm{dL}$ ), se pudo demostrar la existencia de una estrecha relación entre estas dos variables $(\mathrm{p}<0,01)$.

Tabla 2. Frecuencias de genotipos y alelos polimorfismo S19Wen la población analizada; afectados por hipertrigliceridemia como no afectados.

\begin{tabular}{lccccc}
\hline & Genotipo, n (\%) & & \multicolumn{2}{c}{$\begin{array}{c}\text { Frecuencias } \\
\text { Alélicas, } \mathbf{n}(\%)\end{array}$} \\
\hline $\begin{array}{l}\text { Genotipo } \\
\text { de S19W }\end{array}$ & CC & CG & GG & Alelo C & Alelo G \\
$\begin{array}{l}\text { Afectados } \\
(\mathbf{n = 1 5 9 )}\end{array}$ & $6(3.8)$ & $38(23.9)$ & $115(72.3)$ & $31(0.11)$ & $249(0.89)$ \\
$\begin{array}{l}\text { No } \\
\text { afectados } \\
(\mathbf{n = 2 4 1 )}\end{array}$ & $3(1)$ & $59(24.5)$ & $179(74.3)$ & $35(0.08)$ & $387(0.92)$ \\
Valor de p & 0,32 & 0,03 & $<0,01$ & 0,62 & $<0,01$ \\
\hline
\end{tabular}

EHW Afectados: chi $^{2}=1.534 \mathrm{p}=0,22$

EHW No afectados: $\mathrm{chi}^{2}=0.583 \mathrm{p}=0,45$

EHW en el grupo total bajo la H0: de EHW en casos dado que los controles están en EHW, $\operatorname{chi}^{2}(2)=2.168$ p $=0.3382$.

\section{DISCUSIÓN}

Las enfermedades cardiovasculares representan el $29,6 \%$ del total de muertes en Colombia ${ }^{1}$, siendo las enfermedades isquémicas cardíacas la principal causa de estas muertes con un $8,16 \%$, convirtiéndose en 
un problema de salud pública con carga económica para el Sistema Nacional de Salud. Las causas de las ECV son multifactoriales por tanto la prevención y el manejo clínico requiere de la identificación y control tanto de factores genéticos como medio ambientales responsables de manera directa o indirecta de su origen y progresión.

Los recientes avances en el estudio del componente genético de la ECV han contribuido a conocer mejor la historia natural de la enfermedad, y han fortalecido el interés sobre el análisis de los posibles genes implicados en su desarrollo, las variantes polimórficas de estos genes y su potencial como marcador de riesgo o de protección.

La asociación entre la hipertrigliceridemia, enfermedad cardiovascular y mortalidad ha sido controversial ${ }^{17,19}$. Diversos mecanismos se han propuesto para sustentar la hipertrigliceridemia como un factor de riesgo para ECV, entre los que se cuenta su asociación con niveles bajos de cHDL, el incremento en las cLDL pequeñas y densas y la insulino resistencia periférica ${ }^{20}$; adicionalmente la hipertrigliceridemia incrementa la producción de citoquinas pro inflamatorias, fibrinógeno y proteínas de la coagulación, fuertemente asociadas al aumento del riesgo de ECV ${ }^{21,22}$. La APOA5, descrita en $2001^{6}$, alcanza concentraciones plasmáticas de $0,15 \mu \mathrm{gr} /$ $\mathrm{mL}$, y tienen un papel relevante en el metabolismo de los TAG. El Berkeley Lipid Study Population demostró que el polimorfismo S19W (Ser19Ter) de la APOA5 es tres veces más frecuente en varones y mujeres blancas con niveles elevados de triglicéridos al comparar con otras poblaciones similares ${ }^{10}$.

El estudio mostró que, en población colombiana, originada ésta en la mezcla de componente caucásico, negro, oriental y nativo, el genotipo GG de la ApoA5 es el más frecuente, tanto en afectados como no afectados, encontrándose en el $73,5 \%$ de sujetos analizados. El genotipo CC presentó la frecuencia más baja en ambas poblaciones, afectados y no afectados por hipertrigliceridemia (2,2\%). Estos resultados no son similares a los encontrados en otras poblaciones ${ }^{23-27}$. Penachio reportó en norteamericanos e hispanos una frecuencia para el alelo mutado (C) del 15\%, para caucásicos del $6 \%$, para africanos y para americanos del $7 \%{ }^{14}$. Ruiz, et al. informaron una frecuencia de $10,02 \%$ para el polimorfismo Ser19Trp (S19W), en población china, Malaya e hindú, $0,1 \%, 1 \%$ y $3 \%$ respectivamente. Andrade reportó que en poblaciones brasileras con componente mestizo la frecuencia del polimorfismo fue de $13,7 \%{ }^{28}$, resultados que no coinciden con los obtenidos en este trabajo $(73,26 \%)$, sin embargo, concuerdan con los datos reportados por el NCBI en una población de Estados Unidos (ESP) con 87,4\% ${ }^{29}$.

La población estudiada se encontró en equilibrio de Hardy Weinberg, lo que permite inferir que no estaba influenciada por factores externos como deriva genética, mutación o consanguinidad de los individuos ${ }^{30}$.

Con relación a la distribución del polimorfismo S19W (Ser19Ter) de la APOA5 en el grupo de sujetos afectados con hipertrigliceridemia, se encontró que la forma homocigota GG y la heterocigota GC presentaron valores significativos $(p<0,01$ y $p=0,03$, respectivamente), resultado que concuerda con los hallazgos observados en otras poblaciones. Andrade demostró que la presencia del polimorfismo Ser19Trp del Gen de APOA5 influye significativamente en los niveles de triglicéridos y es un factor de riesgo independiente para desarrollar enfermedad coronaria ${ }^{28}$.

La importancia desde el punto de vista funcional del polimorfismo (S19W) Ser19Trp, radica en el cambio de un amino ácido hidrofílico, serina, por uno hidrofóbico, triptófano, en el péptido señal, dificultando su translocación al retículo endoplásmico, lo que sugiere que esto provoca un cambio funcional ${ }^{31}$. Todo parece indicar que la APOA5 actúa como un control para la secreción de TAG por parte del hígado ${ }^{32,33}$. La dificultad que este polimorfismo provoca en la translocación de la proteína, reduce su secreción por parte del retículo endoplásmico, disminuyendo su disponibilidad y por tanto su asociación a las lipoproteínas ricas en TAG, lo que deprime su papel regulador en el crecimiento y depuración de las VLDL y quilomicrón, que se reflejaría en la producción de VLDL excesivamente cargadas de TAG, lo que explicaría su asociación con los altos niveles plasmáticos de $\mathrm{TAG}^{34-36}$.

Los hallazgos alcanzados en este trabajo nos obligan a seguir en la búsqueda de otros posibles marcadores de riesgo que permitan detectar tempranamente a los potenciales candidatos a una hipertrigliceridemia, de manera tal que se logre realizar una intervención temprana en su estilo de vida, mejorando la calidad de la misma, y quizás lograr aumentar su expectativa de vida.

Dentro de las limitaciones de este trabajo cabe señalar que se trata de un primer abordaje de los aspectos genéticos del metabolismo de los TAG, analizando un solo polimorfismo de un gen cuyo estudio hoy en día en otras poblaciones, es fuente de nuevas publicaciones en el tema, con el objetivo claro de conocer mejor a 
la población afectada, de impactar en nuevas pruebas diagnósticas especialmente preventivas, siempre con el ánimo de llegar en un futuro a un número mayor de individuos involucrados. Las más recientes publicaciones muestran no solo un aumento sustancial en el número de sujetos analizados, sino como la relación existente entre diversos productos del metabolismo graso y un número creciente de marcadores genéticos de protección o riesgo, siempre con miras a tener herramientas diagnósticas predictores para selección de candidatos a campañas de prevención.

\section{CONFLICTO DE INTERÉS}

Los autores declaramos que no tenemos ningún conflicto de interés.

\section{CONSIDERACIONES ETICAS}

El presente estudio, fue avalado por el comité de ética de la Facultad de Salud de la Universidad Industrial de Santander según acta $\mathrm{N}^{\circ} 18$ del 11 de octubre del 2010; es considerado de riesgo mínimo según resolución 8430 de 1993 del ministerio de salud, y los datos utilizados son de carácter anónimo para proteger la confidencialidad.

\section{REFERENCIAS}

1. Análisis de la situación de salud, Colombia 2013. Ministerio de Salud y Protección Social; Pág. 3750: 2014.

2. Wang Q. Molecular genetics of coronary artery disease. Curr Opin Cardiol. 2005; 20(3): 182-188.

3. Cullen P. Evidence that triglycerides are an independent coronary heart disease risk factor. Am J Cardiol. 2000; 86(9): 943-949.

4. Heller DA, de Faire U, Pedersen NL, Dahlen G, McClearn GE. Genetic and environmental influences on serum lipid levels in twins. N Engl J Med. 1993; 328: 1150-1156. DOI: 10.1056/ NEJM199304223281603.

5. Korn ED. Clearing factor, a heparin-activated lipoprotein lipase. I. Isolation and characterization of the enzyme from normal rat heart. J Biol Chem. 1955; 215(1); 1-14.

6. Pennacchio LA, Olivier M, Hubacek JA, Cohen JC, Cox DR, Fruchart JC, et al. An apolipoprotein influencing triglycerides in humans and mice revealed by comparative sequencing. Science. 2001; 294(5540): 169-173. DOI: 10.1126/science.1064852.

7. Van der Vliet HN, Sammels MG, Leegwater AC, Levels JH, Reitsma PH, Boers W, et al.
Apolipoprotein A-V: a novel apolipoprotein associated with an early phase of liver regeneration. J Biol Chem. 2001; 276(48): 44512-44520. DOI:10.1074/jbc.M106888200.

8. Ong KL, Jiang CQ, Liu B, Jin YL, Tso AW, Tam $\mathrm{S}$, et al. Association of a genetic variant in the apolipoprotein A5 gene with the metabolic syndrome in Chinese. Clin Endocrinol (Oxf). 2011; 74(2): 206-213. DOI: 10.1111/j.1365-2265.2010.03899.x.

9. Henneman P, Schaap FG, Havekes LM, Rensen PC, Frants RR, van Tol A, et al. Plasma apoAV levels are markedly elevated in severe hypertriglyceridemia and positively correlated with the APOA5 S19W polymorphism. Atherosclerosis. 2007; 193(1): 129134. DOI: $10.1016 / \mathrm{j}$.atherosclerosis.2006.05.030.

10. Pennacchio LA, Olivier M, Hubacek J, Krauss RM, Rubin EM, Cohen JC. Two independent apolipoprotein A5 haplotypes influence human plasma triglyceride levels. Hum Mol Genet. 2002; 11(24): 3031-3038.

11. Evans D, Bode A, Lippe G, Beil FU, Mann WA. Cerebrovascular atherosclerosis in type III hyperlipidemia is modulated by variation in the apolipoprotein A5 gene. Eur J Med Res. 2011; 16(2): 79-84.

12. Sóter MO, Gomes KB, Fernandes AP, Carvalho Md, Pinheiro PS, Bosco AA, et al. -1131T C and SW19 polymorphisms in APOA5 gene and lipid levels in type 2 diabetic patient. Mol Biol Rep. 2012; 39(7): 7541-7548. DOI: 10.1007/s11033-012-1588-X.

13. Olivier M, Wang X, Cole R, Gau B, Kim J, Rubin EM, et al. Haplotype analysis of the apolipoprotein gene cluster on human chromosome 11. Genomics. 2004; 83(5): 912-923. DOI: 10.1016/j.ygeno.2003.11.016.

14. Kisfali P, Mohas M, Maasz A, Hadarits F, Marko L, Horvatovich K, et al. Apolipoprotein A5 IVS3 + 476A allelic variant associates with increased triglyceride levels confers risk for development of metabolic syndrome in Hungarians. Circ J. 2008; 72(1): 40-43.

15. Singmann P, Baumert J, Herder C, Meisinger C, Holzapfel C, Klopp N, et al. Gene-gene interaction between APOA5 and USF1: two candidate genes for the metabolic syndrome. Obes Facts. 2009; 2(4): 235-242. DOI: 10.1159/000227288.

16. Excoffier L, Lischer HE. Arlequin suite ver 3.5: a new series of programs to perform population genetics analyses under Linux and Windows. Mol Ecol Resour. 2010; 10(3): 564-567. DOI: 10.1111/J.1755-0998.2010.02847.X.

17. Miller M, Stone NJ, Ballantyne C, Bittner V, Criqui $\mathrm{MH}$, Ginsberg HN, et al. Triglycerides and cardiovascular disease: a scientific statement from the American Heart Association. Circulation. 
2011; 123(20): 2292-2333. DOI: http://dx.doi. org/10.1161/CIR.0B013E3182160726.

18. Nordestgaard BG, Varbo A. Triglycerides and cardiovascular disease. Lancet. 2014; 384(9943): 626-635. DOI: http://dx.doi.org/10.1016/S01406736(14)61177-6.

19. Guérin M, Le Goff W, Lassel TS, Van Tol A, Steiner G, Chapman MJ. Atherogenic role of elevated CE transfer from HDL to VLDL (1) and dense LDL in type 2 diabetes: impact of the degree of triglyceridemia. Arterioscler Thromb Vasc Biol. 2001; 21(2): 282-288.

20. Ginsberg HN. New perspectives on atherogenesis: role of abnormal triglyceride-rich lipoprotein metabolism. Circulation. 2002; 106(16): 21372142. DOI: 10.1161/01.CIR.0000035280.64322.31

21. Brunzell JD: Clinical practice. Hypertriglyceridemia. N Engl J Med. 2007; 357: 1009-1017. DOI:10.1056/ NEJMcp070061.

22. Lee KW, Ayyobi AF, Frohlich JJ, Hill JS. APOA5 gene polymorphism modulates levels of triglyceride, HDL cholesterol and FER(HDL) but is not a risk factor for coronary artery disease. Atherosclerosis. 2004: 176(1): 165-172. DOI: 10.1016/j.atherosclerosis.2004.04.024.

23. Ajjemami M, Ouatou S, Charoute H, Fakiri M, Rhaissi $\mathrm{H}$, Benrahma $\mathrm{H}$, et al. Haplotype analysis of the Apolipoprotein A5 gene in Moroccan patients with the metabolic syndrome. J. Diabetes Metab Disord. 2015; 14: 29. DOI: 10.1186/S40200-0150160-3.

24. De Castro-Orós I, Cenarro A, Tejedor MT , BailaRueda L , Mateo-Gallego R, Lamiquiz-Moneo I, et al. Common genetic variants contribute to primary hypertriglyceridemia without differences between familial combined hyperlipidemia and isolated hypertriglyceridemia. Circ Cardiovasc Genet. 2014; 7(6): 814-821. DOI: 10.1161/ circgenetics.114.000522.

25. Li S, Hu B, Wang Y, Wu D, Jin L, Wang X. Influences of APOA5 variants on plasma triglyceride levels in Uyghur population. PLoS One. 2014; 9(10): e110258. DOI: 10.1371/journal.pone.0110258.

26. Mattei J, Demissie S, Tucker KL, Ordova JM. Apolipoprotein A5 polymorphisms interact with total dietary fat intake in association with markers of metabolic syndrome in Puerto Rican older adults. J Nutr. 2009; 139(12): 2301-2308. DOI: 10.3945/ jn.109.109900.

27. Ahituv N, Akiyama J, Chapman-Helleboid A, Fruchart J, Pennacchio LA. In vivo characterization of human APOA5 haplotypes. Genomics. 2007; 90(6): 674-679. DOI:10.1016/j.ygeno.2007.08.003.
28. De Andrade FM, Maluf SW, Schuch JB, Voigt F, Barros AC, Lucatelli JF, et al. The influence of the S19W SNP of the APOA5 gene on triglyceride levels in southern Brazil: Interactions with the APOE gene, sex and menopause status. Nutr Metab Cardiovasc Dis. 2011; 21(8): 584-590. DOI: 10.1016/j.numecd.2009.12.013.

29. Hsieh MC, Lin KD, Tien KJ, Tu ST, Hsiao JY, Chang SJ, et al. Common polymorphisms of the peroxisome proliferator-activated receptor $-\gamma$ (Pro12Ala) and peroxisome proliferator-activated receptor- $\gamma$ coactivator-1 (Gly482Ser) and the response to pioglitazone in Chinese patients with type 2 diabetes mellitus. Metabolism. 2010; 59(8): 1139-1144. DOI: 10.1016/j.metabol.2009.10.030.

30. Hartl D, Clark AC. Genetic and phenotipic variation. In Principles of population genetic. Sinauer Associates Publishers Sunderland. 4 ${ }^{\mathrm{a}}$ Ed. Massachusetts: Sinauer associates; 2007: p $4-38$.

31. Talmud PJ, Palmen J, Putt W, Lins L, Humphries SE. Determination of the functionality of common APOA5 polymorphisms. J Biol Chem. 2005; 280(31): 28215-28220. DOI: 10.1074/jbc.M502144200.

32. Schaap FG, Rensen PC, Voshol PJ, Vrins C, van der Vliet HN, Chamuleau RA, et al. ApoAV reduces plasma triglycerides by inhibiting very low density lipoprotein-triglyceride (VLDL-TG) production and stimulating lipoprotein lipase-mediated VLDLTG hydrolysis. J Biol Chem. 2004: 279(27): 2794127947. DOI: $10.1074 / \mathrm{jbc} . \mathrm{M} 403240200$.

33. Talmud PJ, Hawe E, Martin S, Olivier M, Miller GJ, Rubin EM, et al. Relative contribution of variation within the APOC3/A4/A5 gene cluster in determining plasma triglycerides. Hum Mol Genet. 2002; 11(24): 3039-3046. DOI: 10.1093/ $\mathrm{hmg} / 11.24 .3039$.

34. Lookene A, Beckstead JA, Nilsson S, Olivecrona G, Ryan RO. Apolipoprotein A-V heparin interactions: implications for plasma lipoprotein metabolism. J Biol Chem. 2005: 280(27); 25383-25387. DOI: 10.1074/jbc.M501589200.

35. Nilsson SK, Lookene A, Beckstead JA, Gliemann J, Ryan RO, Olivecrona G. Apolipoprotein A-V interaction with members of the low density lipoprotein receptor gene family. Biochemistry. 2007; 46(12): 3896-3904. DOI: 10.1021/bi7000533.

36. Beigneux AP, Davies BS, Gin P, Weinstein MM, FarberE, QiaoX, etal.Glycosylphosphatidylinositolanchored high density lipoprotein-binding protein 1 plays a critical role in the lipolytic processing of chylomicrons. Cell Metab. 2007: 5(4): 279-291. DOI:10.1016/j.cmet.2007.02.002. 